\title{
A Rule-Based Exclusion Method for Tolerance Specification of Revolving Components
}

\author{
Qijian Zhao ${ }^{\mathrm{a}, \mathrm{c}}$, Tukun $\mathrm{Li}^{\mathrm{c}}$, Yanlong $\mathrm{Cao}^{\mathrm{a}, \mathrm{b} *}$, Jiangxin Yang ${ }^{\mathrm{b}}$, Xiangqian Jiang ${ }^{\mathrm{c}}$ \\ ${ }^{a}$ State Key Laboratory of Fluid Power and Mechatronic Systems, College of Mechanical Engineering, Zhejiang University, \\ Hangzhou, 310027, China \\ ${ }^{b}$ Key Laboratory of Advanced Manufacturing Technology of Zhejiang Province, College of Mechanical Engineering, Zhejiang \\ University, Hangzhou, 310027, China \\ ${ }^{c}$ EPSRC Future Advanced Metrology Hub, Centre for Precision Technologies, School of Computing and Engineering, \\ University of Huddersfield, Huddersfield, HDI 3DH, UK \\ *Corresponding author. Tel.:+86-571-87953198; fax: +86-571-87951145.E-mail address: sdcaoyl@zju.edu.cn.
}

\begin{abstract}
:
This paper proposes a novel method for tolerance specification on revolving components. The revolving parts are widely used and functionally important. An exclusion approach is introduced to construct a tolerance specification method. A functional analysis tool is developed to select features to be specified and to generate their Datum Reference Frame (DRF). A set of rules are modelled to create suitable specification schemes. The independent axiom and entropy theory are applied for further specification refinement. A software application is developed, and an RV reducer is used as a case study. A comparison with other specification methods is undertaken.
\end{abstract}

Key Words: Computer-Aided Tolerancing (CAT); Tolerance specification; Exclusion approach; Datum Reference Frame (DRF); Geometrical tolerances; Revolving part.

\section{Introduction}

Tolerancing plays an essential role in the management of the geometrical variation of a product from conceptual design to final product ${ }^{1,2,3}$. With the trend toward digital manufacturing and intelligent manufacturing ${ }^{4}$, the related international standard is evolving to include an enriched description of both a real product and its digital twin ${ }^{5}$. This enrichment helps the engineers ensure a given geometrical variation of a product throughout its life-cycle. On another hand, this enrichment increases the difficulty in the development of tolerancing specification for the engineer. To this end, some software packages have been developed to assist the designer (detailed in Section 2).

This paper focuses on the tolerance design of revolving components such as shafts, rollers, and so on. They are widely used in mechanical products and play an essential role in their functional performance.

Computer-Aided Tolerancing (CAT) ${ }^{6}$ was introduced in the 1980s. Tolerance design ${ }^{7}$ often includes three tasks: tolerance specification, tolerance allocation and tolerance analysis. Tolerance specification also consists of three sub-tasks ${ }^{7}$ : (a) determination of a DRF; (b) selection of the tolerance, (c) assignment of a tolerance principle. This paper focuses on sub-task (a) and (b), as 
sub-task (c) is highly dependent on an individual engineer's judgments, a process which is currently not sufficiently quantified to allow it to be captured in this way ${ }^{8}$.

To develop a tolerance specification, researchers have previously proposed and developed methods classified as reasoning approaches (see the review in Section 2). In this paper, we propose a new approach for developing a tolerance specification, namely one involving a principle of rule-based exclusion (RBE). Section 3 details the framework and Section 4 presents the implementation of the RBE method. The assessment of its performance is in Section 5, which is followed by the conclusions and future work.

\section{Methodology}

\subsection{Literature review}

1) Technologically and Topologically Related Surfaces (TTRS) method

Clemént et al. ${ }^{9}$ proposed the TTRS method which is probably the first computer-aided tolerance specification model. The TTRS method introduced the Minimum Geometric Datum Element (MGDE) model to classify the features of a part and to apply a given set of rules. The correct application of the TTRS rules is reliant on having an experienced user making the correct judgement calls.

\section{2) Rule-based reasoning methods}

The rule-based methods have been developed to reduce the outcomes dependence on a designer's experience. The rules (in the form of a list of conditions) can be constructed from mathematical theories, assembly relationships and/or empirical knowledge. Rule-based methods are widely used in many fields (e.g. assembly lines ${ }^{10}$, design evaluation ${ }^{11}$ and so forth).

For tolerance specification, several mathematical theories have been developed in order to build the rules or reasoning matrix. Zhang et al. ${ }^{12}$ introduced the Polychromatic Set Theory, while Zhong ${ }^{13}$ and Qin ${ }^{14}$ developed ontology-based representation methods, and $\mathrm{Hu}$ et al ${ }^{15}$ proposed a Variational Geometric Constraints Network-based method. These methods are less dependant on the users' experience, however, these models are relatively complicated.

Other methods have been developed according to the assembly positioning constraints. Anselmetti et al. ${ }^{16}$ proposed a positioning-table method which they have implemented in a CAD system ${ }^{17}$, while Cao et al. ${ }^{18}$ applied graph theory to validation algorithms. Those methods do not, however, take into account the limitations on a measurement method in certain conditions.

Finally, there are empirical rule-based methods such as that by Armillotta ${ }^{19}$, and Haghighi et al. ${ }^{20}$ These methods consider both the manner of assembly and inspection, though sometimes their rules may be too rigid to allow them to be successfully applied.

3) Case-based reasoning method

Some case-based reasoning methods and machine learning method ${ }^{21}$ have been introduced. Cao et al. ${ }^{22}$ proposed a statistical learning based method for datum selection. Qin et al..$^{23}$ developed an ontology-supported case-based reasoning approach. These show the possibility of creating a tolerance specification via machine learning, but currently, their successful application is limited due to the lack of sufficient data to train the algorithms.

Table 1 is a comparison of the above methods. Using this comparison we have developed a method taking into account the following considerations: 1) the manner of assembly and 
inspection, 2) be ISO standard ${ }^{1}$ compatible, 3) use a rule-based method, and 4) be easy to apply.

In the $15^{\text {th }}$ CIRP conference on CAT, further works on tolerance specification were presented, including that by Zbigniew ${ }^{24}$, Stefan ${ }^{25}$ and Stanislao ${ }^{26}$, that can be classified as the reasoning approach as well.

Table 1 Comparison of current tolerance specification methods

\begin{tabular}{|c|c|c|c|c|}
\hline $\begin{array}{c}\text { Tolerance specification } \\
\text { methods }\end{array}$ & $\begin{array}{l}\text { Factors for } \\
\text { specification }\end{array}$ & Standards & $\begin{array}{l}\text { Model or } \\
\text { method }\end{array}$ & $\begin{array}{c}\text { Difficulty of } \\
\text { Implementation }\end{array}$ \\
\hline Clemént $^{9}$ & Geometry, position. & ISO & MGDE \& rules & Simple \\
\hline Haghighi ${ }^{20}$ & $\begin{array}{l}\text { Geometry, position, } \\
\text { assembly. }\end{array}$ & ASME & Rules & Simple \\
\hline Armillotta $^{19}$ & $\begin{array}{l}\text { Geometry, position, } \\
\text { assembly, process. }\end{array}$ & ASME & $\begin{array}{c}\text { Reasoning table } \\
\quad \& \text { rules }\end{array}$ & Simple \\
\hline Anselmetti ${ }^{17}$ & $\begin{array}{l}\text { Geometry, position, } \\
\text { assembly. }\end{array}$ & ASME & $\begin{array}{l}\text { Positioning } \\
\text { table \& rules }\end{array}$ & Medium \\
\hline Zhang $^{12}$ & $\begin{array}{l}\text { Geometry, position, } \\
\text { assembly. }\end{array}$ & ISO & $\begin{array}{l}\text { Polychromatic } \\
\text { sets \& relation } \\
\text { matrix }\end{array}$ & Difficult \\
\hline Qin $^{23}$ & $\begin{array}{l}\text { Geometry, position, } \\
\text { assembly, successful } \\
\text { cases. }\end{array}$ & ISO & $\begin{array}{c}\text { Ontology \& } \\
\text { calculation of } \\
\text { similarity }\end{array}$ & Difficult \\
\hline
\end{tabular}

\subsection{The proposed method}

Table 2 shows diagrammatically both the reasoning approach and the exclusion approach. All the methods listed in section 2.1 can be classified as reasoning approaches. The process taken by the reasoning approach is to develop the tolerances from the geometrical requirements of the objects specification, as shown in table 2 . The rules, tables, matrixes or cases are applied to select geometrical tolerances directly by applying the following steps (See example in Table 2): 1) a object, e.g. a cylinder, is required to be toleranced; 2) the rule is followed, e.g. that the cylinder is specified by its cylindricity; and 3) tolerancing is applied, e.g. cylindricity is selected.

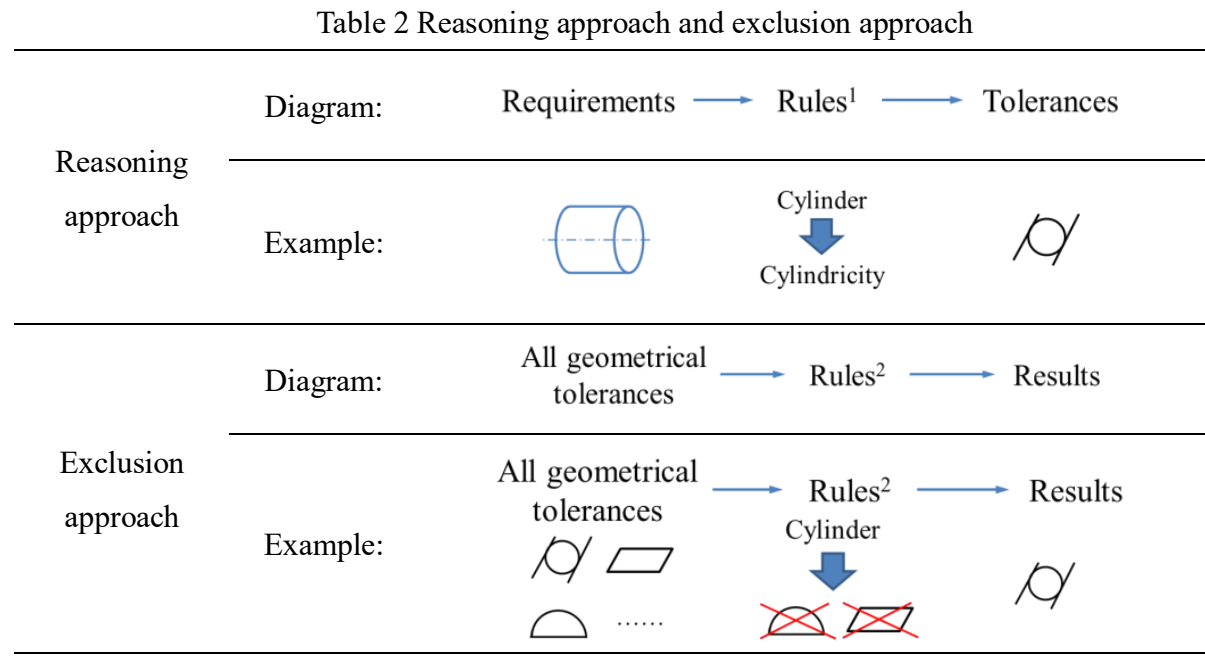

Note $^{1}$ : The Rules are the mapping from the functional requirement of the feature to the geometrical tolerances.

Note $^{2}$ : The Rules are the mapping from the geometrical tolerances to the final tolerance specification 
scheme.

An exclusion approach (see Table. 2) is introduced for tolerance specification in this paper. This approach has been widely applied in many fields, e.g. biotechnology ${ }^{27}$, robotics ${ }^{28}$. This method uses three steps to develop the tolerance (see example in Table 2): 1) to list all possible geometrical tolerances for a feature; 2) to eliminate the tolerance items according to the rules; e.g. everything except cylindricity; 3) to use the remaining tolerance items as the outcome of the tolerance specification.

In general, the exclusion approach will provide either the same number, or more, possible solutions than the reasoning approach, though it may take longer to calculate that the traditional method if the number of tolerance items is large. We will develop the RBE method in the following sections with the above considerations.

\section{The development of Rule-Based Exclusion (RBE) method}

The character of "revolving" is utilized to model rules for datum selection. The factors of geometry, position and assembly are modelled as fixed constraints by rigid rules. Axiomatic Design (AD) theory ${ }^{29}$ and entropy theory ${ }^{30}$ are used for refinement. Fig. 1 illustrates the flow of the RBE method with the key steps being shown in bold, these will be documented in subsections 3.1-3.2.

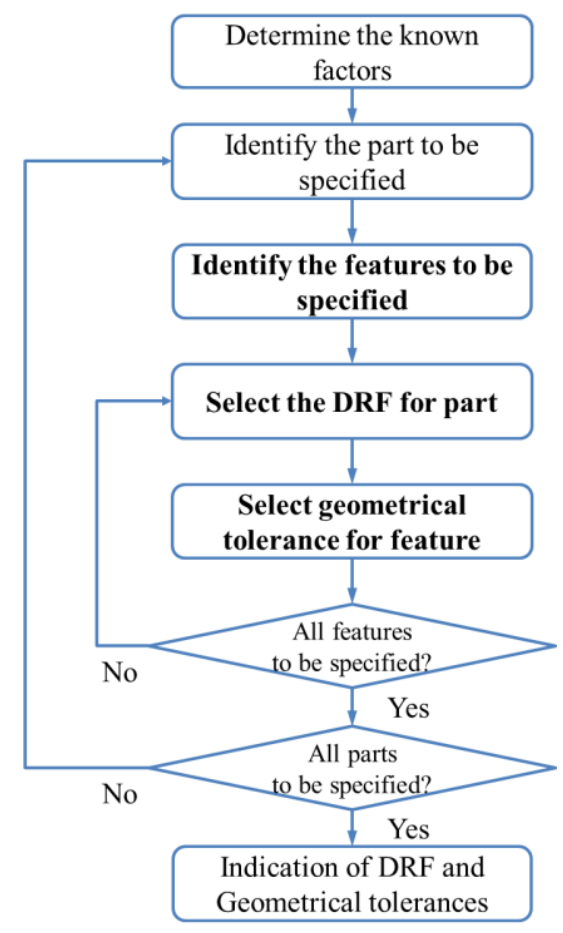

Fig. 1 Specification flow of the RBE method

To identify the features to be specified, information about the parts function ${ }^{17}$, how it is joined ${ }^{19}$ and its assembly ${ }^{23}$ is required. The following should be specified: (1) joined and assembly features; (2) any functional features; (3) any datum features.

\subsection{Formulation of Datum Reference Frame (DRF)}

When determining the DRF, there are several significant factors ${ }^{31}$ : 
1. Geometrical size and accuracy grade: The largest features, and/or those having a higher accuracy grade are often most suitable to be selected as a datum ${ }^{32}$.

2. Geometrical type: Which determine the orientation of a datum 9 .

3. Inspection method: The datum has to be suitable for measurement.

For the revolving parts, an axis datum and a plane datum should be selected via the following operations: (1) the selection of features in order to derive the central axis together with the plane perpendicular to this axis, and (2) the determination of the primary datum. Rule $\mathbf{1}$ and Rule $\mathbf{2}$ are defined for operation (1) and (2) respectively.

\section{Rule 1: Datum features selection rule}

A feature (i.e. revolving surfaces) is to be selected if the central axis can be derived from it. If several features satisfy this condition, the primary selection is the feature(s) related to supporting parts (such as bearing and support frame).

To derive the planar datum, a plane feature is selected which does not have an assembly relationship with another part. If several features satisfy this condition, then the feature with the largest area is selected.

\section{Rule 2: Primary datum selection rule}

The plane datum feature is selected as a primary datum, if $r_{d}>2$; otherwise, the revolving feature(s) is selected as a primary datum.

Definition 1: The relative size of a datum feature (note as $r_{d}$ ) is the ratio of the length of the revolving feature in the axial direction and length of the plane in the radial direction.

The factor $r_{d}$ is determined according to the related literature ${ }^{19,20}$.

Example 1: The length of the plane in a radial direction, $D$, the length of the revolving feature in the axial direction, $L$, and $r_{d}$ are shown in Fig. 2.

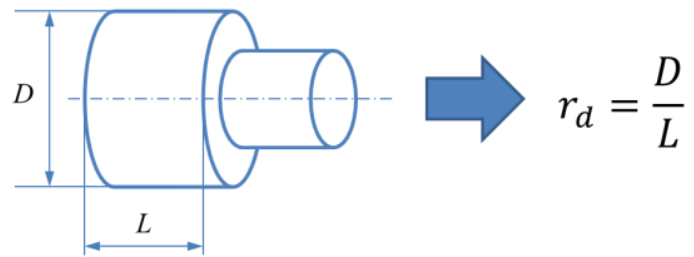

Fig. 2 An illustration of $r_{d}$

\subsection{Geometrical tolerances selection}

Fig. 3 shows the process of tolerance selection. Note that the process may terminate at any step when a unique tolerance scheme is obtained. 


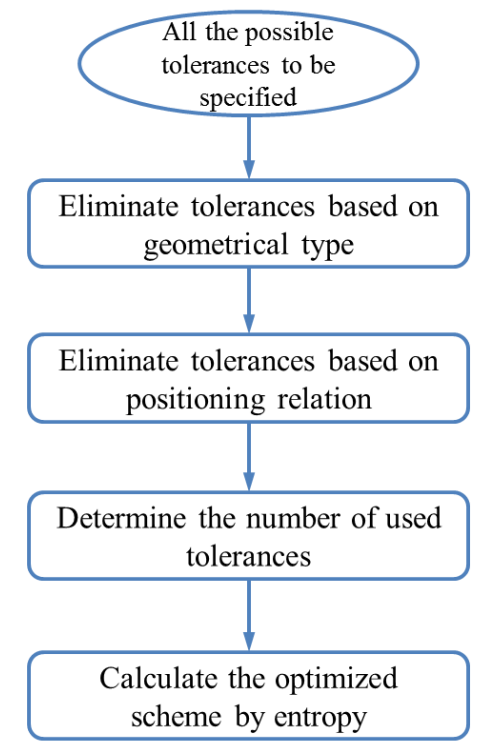

Fig. 3 The process of geometrical tolerances selection

Initially, all possible tolerances are listed. All the geometrical tolerances ${ }^{33}$ and the DPs, along with the symbols used to denote them are listed in Table 3.

Table 3 DPs, symbol and requirement of a datum of each geometrical tolerance

\begin{tabular}{|c|c|c|c|c|}
\hline $\mathrm{DPs}^{1}$ & Geometrical tolerance & Symbol & $\begin{array}{c}\text { The requirement of } \\
\text { datum or not }\end{array}$ & Tolerance type \\
\hline$D P_{1}$ & Straightness & - & No & Form \\
\hline$D P_{2}$ & Flatness & $\square$ & No & Form \\
\hline$D_{3}$ & Roundness & 0 & No & Form \\
\hline $\mathrm{DP}_{4}$ & Cylindricity & d & No & Form \\
\hline$D P_{5}$ & Parallelism & $/ /$ & Yes & Orientation \\
\hline$D P_{6}$ & Perpendicularity & $\perp$ & Yes & Orientation \\
\hline$D P_{7}$ & Angularity & $<$ & Yes & Orientation \\
\hline$D P_{8}$ & Coaxiality & (2) & Yes & Positioning \\
\hline$D P_{9}$ & Symmetry & $=$ & Yes & Positioning \\
\hline$D P_{10}$ & Position & $\phi$ & Yes & Positioning \\
\hline$D P_{11}$ & Circular Runout $^{2}$ & I & Yes & Positioning \\
\hline$D P_{12}$ & Total Runout $^{2}$ & $\angle$ & Yes & Positioning \\
\hline$D P_{13}$ & Concentricity & (a) & Yes & Positioning \\
\hline$D P_{14}$ & Line Profile ${ }^{3}$ & $\frown$ & Yes / No & l \\
\hline$D P_{15}$ & Surface Profile ${ }^{3}$ & $\bigcirc$ & Yes / No & / \\
\hline
\end{tabular}

${ }^{1}$ The design parameters (DPs) are used to describe the task of geometrical tolerances design.

${ }^{2}$ Because the circular and total runout determines the position, they are classified as position tolerance here.

${ }^{3}$ Line and surface profile can be used as form tolerance, orientation tolerance or position tolerance, so the datum requirement is uncertain.

The geometrical type of a feature is an essential characteristic in the selection of tolerancing. Thus, the geometrical type rule is introduced.

\section{Rule 3: Geometrical type rule}

A geometrical tolerance must satisfy the requirements of the feature's geometrical types; otherwise, it will be removed from candidate tolerances. 
The geometrical type of a feature can sometimes determine the geometrical tolerances. The geometrical tolerance for a freeform surface is unique (i.e. $\bigcirc$ ), while the geometrical tolerance for a plane is variable. Table A1 lists the possible geometric tolerances according to the geometrical type ${ }^{9}$ and their definitions ${ }^{1}$.

For some features, i.e. a plane and a revolving surface, there are many possible results. Rule 4 is then applied which is an exclusion operation based on the TTRS method 9 .

\section{Rule 4: Positioning relationship rule}

A geometrical tolerance must fulfil the requirements of the relationship between a feature and the primary datum; otherwise, it will be eliminated from candidate tolerances.

For example, if a feature is a parallel to the primary datum, then $D P_{5}(/ /)$ is employed for control of the orientation, but not $D P_{11}(\perp)$ or $D P_{12}(\angle)$. If a feature is a primary datum, its orientation and position variation would not be specified. Table A2 illustrates different conditions and their corresponding possible geometrical tolerance.

The $\mathrm{AD}$ theory is introduced for further exclusion and selection. The variation requirement is treated as a functional requirement (FR), the geometrical tolerance is described as a design parameter (DP) ${ }^{34}$, and a design matrix is used to model their relationships. Rule 5 and Rule $\mathbf{6}$ are based on the independent axiom and the information axiom.

\section{Rule 5: The independent axiom rule}

The tolerances are organised according to the independent axiom rule if several candidate tolerances exist.

The independent axiom ${ }^{29}$ requires that the design matrix should be triangular or diagonal, in other words: The number of FRs and DPs should be equal, and each FR could be satisfied by adjusting a DP individually or by several DPs orderly.

Let $F R_{\mathrm{R}}$ be the variation requirement of a revolving feature. The variation of a feature can be decomposed into form variation $F R_{1}$, rotation variation $F R_{2}$ and translation variation $F R_{3}$, according to the kinematics ${ }^{35}$ and tolerance analysis method ${ }^{36}$ (See in Fig. 4). Based on the definition of the tolerances, $F R_{1}$ can be controlled by the form tolerance, $F R_{2}$ by the orientation tolerance, and $F R_{3}$ by the position tolerance ${ }^{33}$.

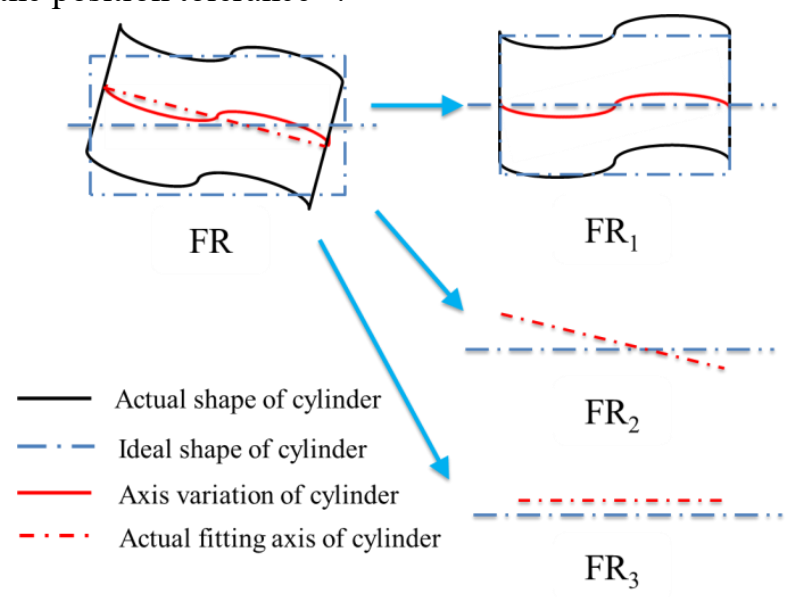

Fig. 4 Decomposition of the variation of the revolving feature

$$
\text { (Cylinder for example) }
$$

Based on Rule 5, three geometrical tolerances are applied to a revolving feature:

$D P$ for $F R_{1}$ of $F R_{\mathrm{R}}$ can be: $D P_{1}(-), D P_{3}(\bigcirc)$ or $D P_{4}(D /)$;

$D P$ for $F R_{2}$ of $F R_{R}$ can be: $D P_{5}(/ /), D P_{6}(\perp)$ or $D P_{7}(\angle)$; 
$D P$ for $F R_{3}$ of $F R_{\mathrm{R}}$ can be: $D P_{8}(\bigcirc), D P_{9}(=), D P_{10}(\phi), D P_{11}(/)$ or $D P_{12}(\angle)$.

Note 1: if a plane is coplanar to the primary datum, or a cylinder coaxial to the primary datum, $F R_{2}$ will not be taken into account.

Note 2: if a plane or cylinder is used as the primary datum, $F R_{2}$ and $F R_{3}$ will not be considered.

If the use of Rule 3 - Rule 5 does not produce a unique result, Rule $\mathbf{6}$ is employed to produce the optimized scheme based on the information entropy ${ }^{30}$.

\section{Rule 6: Entropy rule}

If there are several candidate tolerance schemes, the scheme with the least entropy is selected.

The concept of the entropy is introduced to estimate the cost and efficiency of a geometrical tolerance application. It is defined as follows:

Definition 2: The entropy, $S_{i}$, is

$$
S_{i}=\log _{2} \frac{3 m_{i}}{V_{i}}+\log _{2} \frac{c_{i}}{c_{\min }}
$$

where $c_{i}(i=1,2, \ldots, 15)$, is the geometrical tolerance application, $m_{i}$ is the inspection precision, $V_{i}$ is the maximum accepted variation and $c_{\text {min }}$ is the minimal cost of all DPS

Since the inspection precision has to be three times greater than the accepted variation, the coefficient " 3 " is introduced.

If $3 m_{i}<V$, then let $\log _{2} \frac{3 m_{i}}{V_{i}}=0$.

The entropy of the selection scheme, $S$, is then

$$
S=\sum_{1}^{n} S_{i}
$$

where $n$ is the number of the geometrical tolerances, and $S_{i}$ is a single tolerance of selection scheme. The selection scheme with minimum $S$ would be selected as the optimized scheme.

Note: If the number of optimized schemes with minimum $S$ is more than one, then one of them will be selected randomly for the tolerance specification.

\section{Implementation and Case Study}

\subsection{Implementation}

A software application has been carried out in order to test and verify the RBE method (illustrated in Fig. 5). The selection of geometrical tolerance and indication are implemented by Visual Basic for Application (VBA) in Solidworks. In the Solidworks environment, the geometrical type of feature, size and position relation can be obtained from the CAD model allowing the geometric tolerances selection to be executed. The Solidworks software package provides a DimXpert module, which has an annotation function, which is implemented by calling the API (Application Program Interface) of DimXpert. 


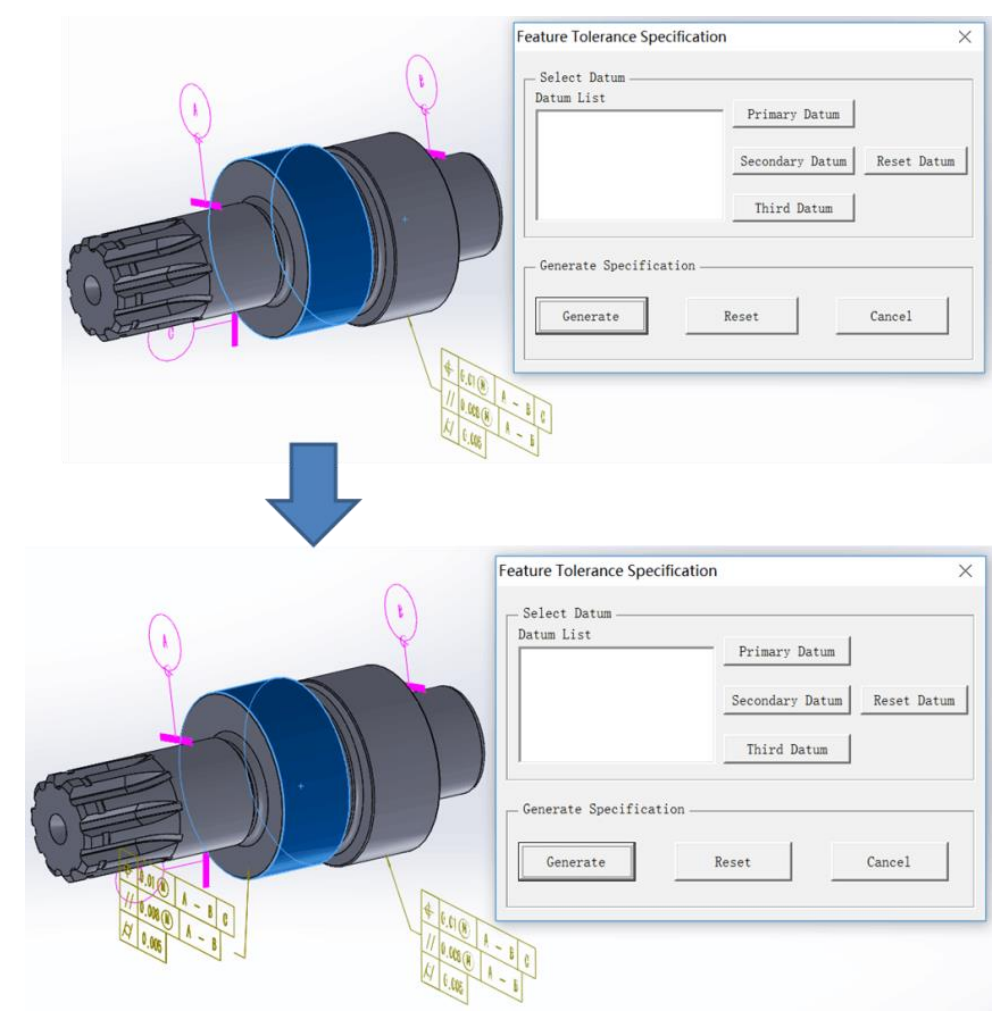

Fig. 5 The tolerance specification program for feature

The current version of API of Solidworks limits the implementation of automatic tolerancing: (1) there is a degree of difficulty in identifying features with multiple surfaces (e.g. a multiple-keys feature); (2) it requires an initial approximation to the value.

\subsection{Case Study}

$\mathrm{An}^{\mathrm{RV}} \mathrm{V}^{37}$ ( $\mathrm{RV}$ is a code of model) reducer mechanism are shown in Fig. 6. We assume that 1) the designer has taken this RV reducer from a sketch ${ }^{38}$; 2) there is no previous case for reference; 3) the machining method ${ }^{39}$ used to make the RV reducer is unknown; and 4) the geometric information, the assembly relationship and the possible inspection method are known.

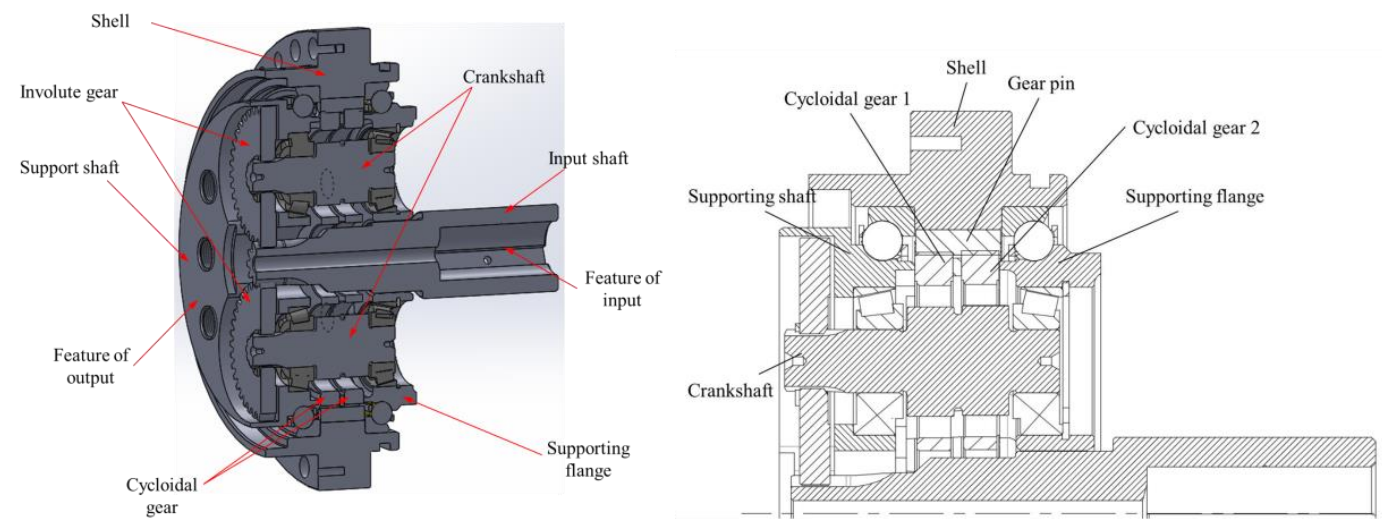

Fig. 6 The primary mechanism of RV reducer

The crankshaft and cycloidal gear used in this paper are shown in Fig. 7, with the features $\mathrm{f} 2, \mathrm{f} 3, \ldots, \mathrm{f} 8 \mathrm{needing}$ to be specified as they have an assembly relationship with other features. 

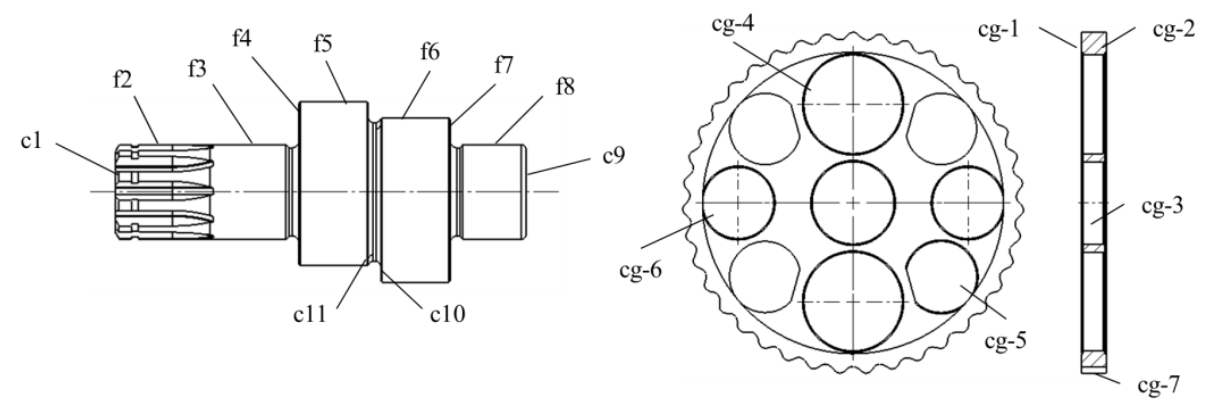

Fig. 7 The features of the crankshaft and cycloidal gear

In this case, the runout measurement device and Coordinate Measuring Machine (CMM) are used for inspection. The effect of temperature is constant during inspection ${ }^{40}$. An estimation of all the geometrical tolerances, $m_{\mathrm{i}}, c_{\mathrm{i}}\left(c_{\mathrm{i}}\right.$ is a relative value) and $V_{\mathrm{i}}$ are listed in Table 4 , which allows Rule 6 to be applied.

Table 4 The entropy of each geometrical tolerance

\begin{tabular}{cccccc}
\hline $\mathrm{DPs}$ & $m_{i} / \mathrm{mm}$ & $c$ & $V_{i} / \mathrm{mm}$ & $S_{i}$ & Tolerance \\
\hline$D P_{1}$ & 0.001 & 1 & 0.003 & 0.6989 & Straightness \\
$D P_{2}$ & 0.001 & 1 & 0.003 & 0.6989 & Flatness \\
$D P_{3}$ & 0.002 & 1 & 0.004 & 1.2839 & Roundness \\
$D P_{4}$ & 0.002 & 1 & 0.005 & 0.9620 & Cylindricity \\
$D P_{5}$ & 0.003 & 1 & 0.008 & 0.8688 & Parallelism \\
$D P_{6}$ & 0.003 & 1 & 0.008 & 0.8688 & Perpendicularity \\
$D P_{7}$ & 0.003 & 1 & 0.009 & 0.6989 & Angularity \\
$D P_{8}$ & 0.004 & 1 & 0.01 & 0.9620 & Coaxiality \\
$D P_{9}$ & 0.004 & 1 & 0.01 & 0.9620 & Symmetry \\
$D P_{10}$ & 0.004 & 1 & 0.01 & 0.9620 & Position \\
$D P_{11}$ & 0.002 & 0.2 & 0.008 & 0 & Circular Runout \\
$D P_{12}$ & 0.004 & 0.2 & 0.01 & 0.2630 & Total Runout \\
$D P_{13}$ & 0.004 & 1 & 0.01 & 0.9620 & Concentricity \\
$D P_{14}$ & 0.004 & 1 & 0.01 & 0.9620 & Line profile \\
$D P_{15}$ & 0.004 & 1 & 0.01 & 0.9620 & Surface profile \\
\hline$A c c o r d i g$ &
\end{tabular}

According to Rule 1, cg-3 and cg-1/cg-2 are selected as the datum features for the cycloidal gear, while $\mathrm{f3}$, $\mathrm{f} 8$ and either $\mathrm{f} 4$ or $\mathrm{f} 7$ are selected for the crankshaft. The DRF selection process is undertaken by applying Rule 2, and the results are illustrated in Fig. 8. 


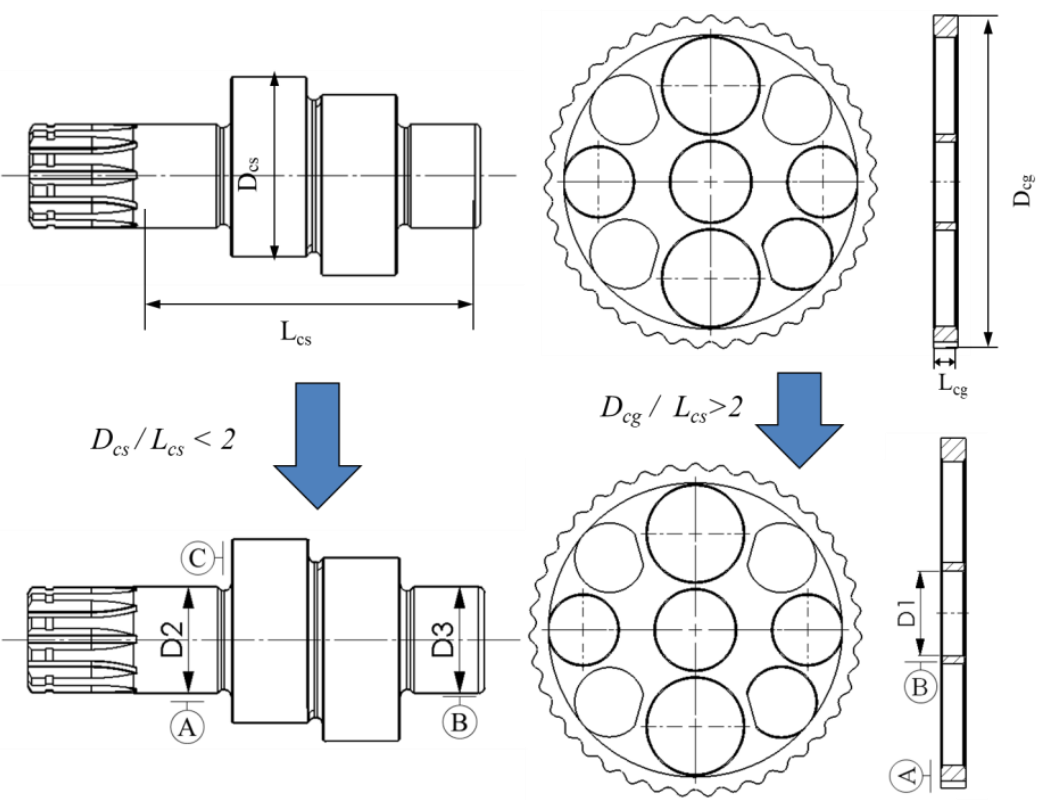

Note: The "A-B" of crankshaft and "A" of cycloidal are the primary datum

Fig. 8 DRF selection process and results

The specification of features $\mathrm{f} 2$ and $\mathrm{f} 7$ are used as an illustration of the geometrical tolerance selection. Feature $\mathrm{f} 2$ is a multiple-keys feature, and according to Rule 3, $D P_{9}(\equiv)$ and $D P_{11}(/)$ should be adopted. Fig. 9 shows the process flow for feature f7.

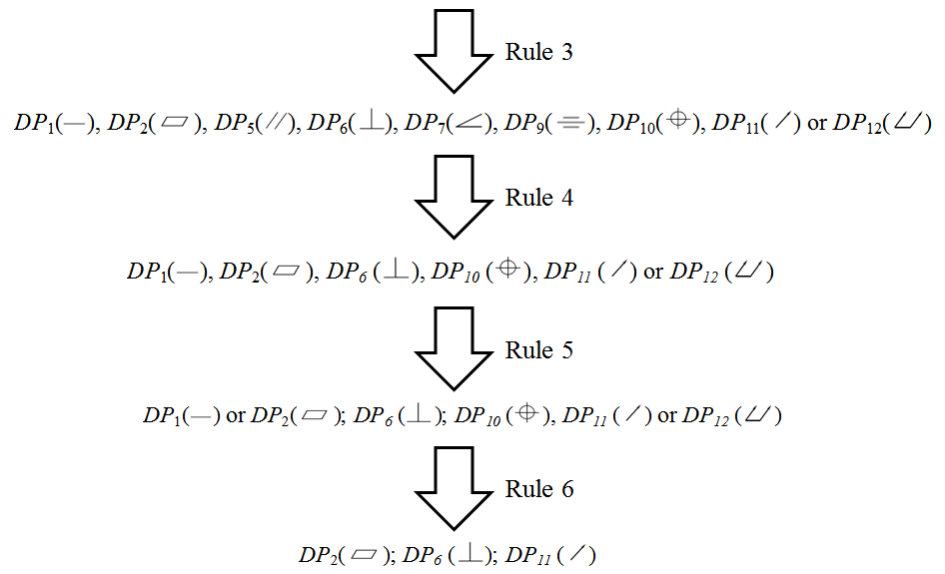

Fig. 9 The specification flow of feature $f 7$

The specification results for the crankshaft and cycloidal gear are shown in Fig. 10. The results of the RBE method, do not conflict with the requirements of the TTRS method, and strictly adhere to the ISO standard ${ }^{1}$. Moreover, the software application produces a specification of each feature takes less than $2 \mathrm{~s}$, which is sufficient for most applications. Therefore, this implementation addresses the common design requirements.

The examples of the crankshaft and cycloidal gear includes a broad range of the typical types of features and positional relationships that would be expected, which indicates that this RBE method is applicable in many situations. 

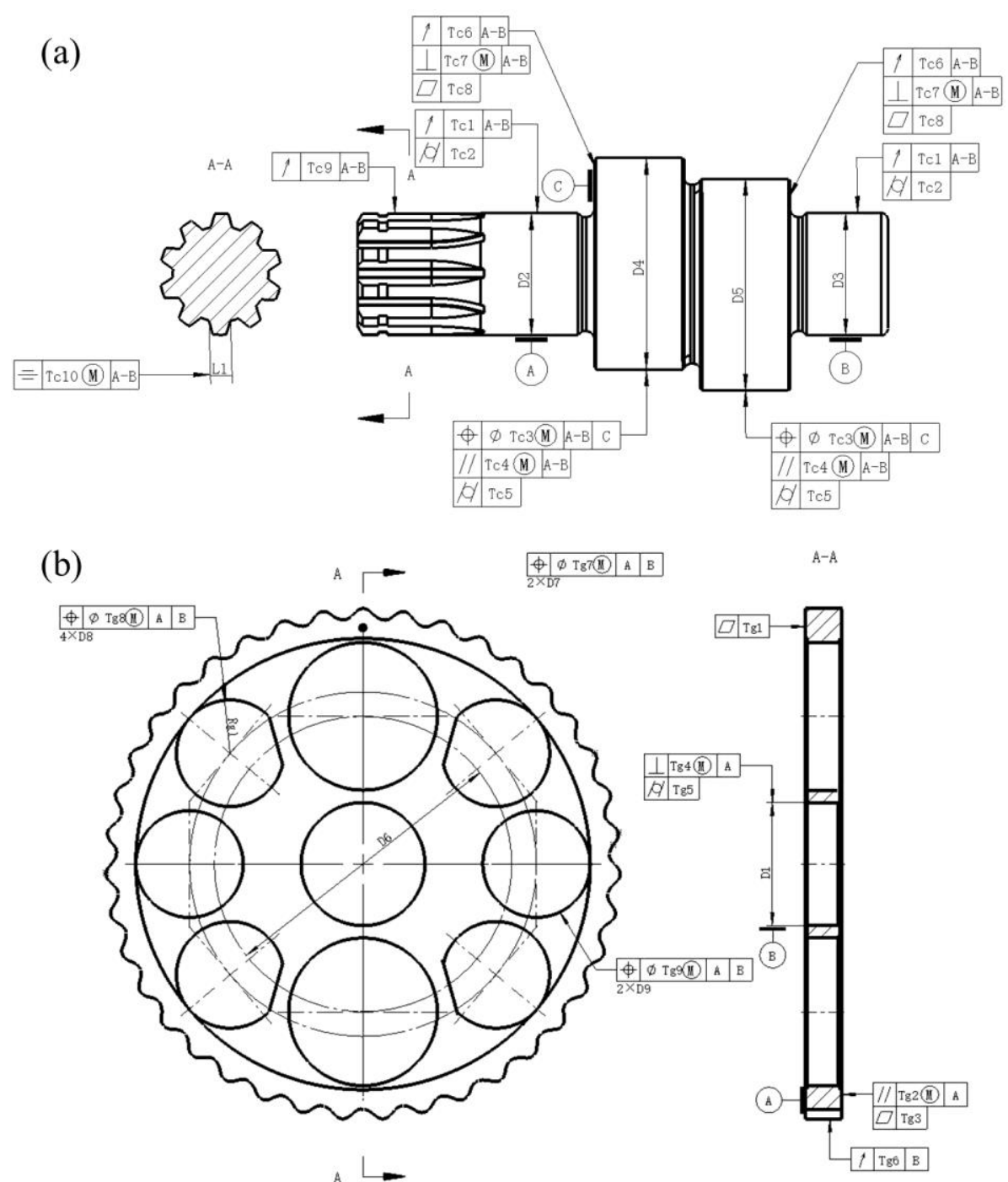

Fig. 10 Tolerance specification result of (a) the crankshaft and (b) the cycloidal gear

A comparison of the RBE method with other tolerance specification methods has been undertaken and the results are shown in Table 5 and 6 .

The results selected by the RBE method are compatible with other methods in Table 5 and 6 , however, the RBE method selects a different DRF for the cycloidal gear. The RBE method selects cg-1 as a primary datum, while the methods of Zhang and Qin select cg-3. Also, the RBE method selects cg-3 as a secondary datum, while the methods of Armillotta and Anselmetti selects cg-4. The different selection of geometric tolerances (due to the different $\mathrm{DRFs}^{41}$ ) is responsible for the selection of either cg-1 or cg-3. 
Table 5 Specification results of the crankshaft

\begin{tabular}{|c|c|c|c|c|c|c|}
\hline No. & F. type & RBE method & Armillotta $^{19}$ & Anselmetti ${ }^{17}$ & Zhang $^{12}$ & Qin $^{23}$ \\
\hline DRF & I & $\mathrm{f} 3 \& \mathrm{f} 8, \mathrm{f} 4$ & $\mathrm{f} 3, \mathrm{f} 4$ & $\mathrm{f} 3, \mathrm{f} 4, \mathrm{f} 5$ & $\mathrm{f} 3 \& \mathrm{f} 8$ & $\mathrm{f} 3 \& \mathrm{f} 8$ \\
\hline $\mathrm{f} 2$ & M. key & $=, \quad /$ & $\phi$ & $\phi$ & $\phi, I$ & $\phi, /$ \\
\hline f3 & Cylinder & $\iota, O$ & - & $\varphi d \pm t / 2 @$ & $\iota, O$ & 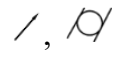 \\
\hline $\mathrm{f} 4$ & Plane & $/, \perp, \square$ & $\perp$ & $\perp$ & 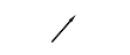 & $\angle$ \\
\hline f5 & Cylinder & $\phi, \|, \alpha$ & $\phi$ & $\phi, / /$ & $\phi, \phi \gamma$ & $\phi, \phi \gamma$ \\
\hline f6 & Cylinder & $\phi, \|, \alpha$ & $\phi$ & $\phi, / /$ & $\phi, \phi$ & $\phi, \alpha$ \\
\hline f7 & Plane & $\nearrow, \perp, \square$ & $\perp$ & $\perp$ & I & $\angle$ \\
\hline f8 & Cylinder & 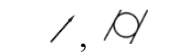 & $\phi$ & $\varphi d \pm t / 2 \circledast$ & 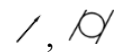 & $\checkmark, O$ \\
\hline
\end{tabular}

Table 6 Specification results of cycloidal gear

\begin{tabular}{|c|c|c|c|c|c|c|}
\hline No. & F. type & RBE method & Armillotta $^{19}$ & Anselmett ${ }^{17}$ & Zhang $^{12}$ & Qin $^{23}$ \\
\hline DRF & l & cg-1, cg-3 & cg-1, cg-4 & cg-1, cg-4 & cg-3, cg-1 & cg-3, cg-1 \\
\hline cg-1 & Plane & $\square$ & $\square$ & $\square$ & / & / \\
\hline $\operatorname{cg}-2$ & Plane & //, $\square$ & // & $\phi, \square$ & ノ & y \\
\hline cg-3 & Cylinder & $\perp, \phi$ & $\phi$ & $\varphi \mathrm{d} \pm \mathrm{t} / 2 \Subset, \phi$ & Q & Q \\
\hline cg-4 & Pattern(2) & $\phi$ & $\phi$ & $\phi, \perp$ & $\phi, \alpha$ & $\phi, \phi \gamma$ \\
\hline cg-5 & Pattern(4) & $\phi$ & $\phi$ & $\phi$ & $\phi$ & $\phi$ \\
\hline cg-6 & Pattern(2) & $\phi$ & $\phi$ & $\phi$ & $\phi$ & $\phi$ \\
\hline cg-7 & Gear & / & $\phi$ & $\phi$ & / & ノ \\
\hline
\end{tabular}

\section{Conclusion and future work}

This paper has documented the design and development of a novel method (namely, the RBE method) for tolerance specification of revolving parts. A review has been undertaken of the existed reasoning approaches. This paper has introduced an exclusion approach for geometrical tolerances selection which considers both the manner of assembly and methods of inspection. An RBE method has been designed and developed, and a case study has been undertaken to illustrate its application. A software package has been developed to implement this method which shows it is relatively easy to apply. A comparison between the results of different methods has been undertaken. This has shown that the RBE method generates the specification which strictly adheres to the ISO standard ${ }^{1}$, and there is no conflict with the requirements of TTRS.

We aim to develop a commercially available software package in the future, however this will require further study and verification work of the extension of the RBE method, and a reliable verification method to assess the tolerance specification.

\section{Acknowledgements}

This research was supported by the National Natural Science Foundation of China (No. 51575484 and U1501248) and Science Fund for Creative Research Groups of the National Natural Science Foundation of China (No. 51521064). The authors would also like to acknowledge the supports by the EPSRC Future Advanced Metrology Hub (Ref. EP/P006930/1). 


\section{References}

1. ISO 1101:2017. Geometrical product specifications (GPS) -- Geometrical tolerancing -- Tolerances of form, orientation, location and run-out.

2. ASME Y14.5: 2004. Dimensioning and Tolerancing.

3. Morse E, Dantan J-Y, Anwer N, et al. Tolerancing: Managing uncertainty from conceptual design to final product. CIRP Annals - Manufacturing Technology 2018; 67: 695-717. DOI: 10.1016/j.cirp.2018.05.009.

4. Anwer, Nabil, Benoît Eynard, Lihong Qiao, et al. Editorial for the Special Issue on 'Smart Manufacturing and Digital Factory'. Proceedings of the Institution of Mechanical Engineers, Part B: Journal of Engineering Manufacture 2019; 233: 1341-1341. DOI:10.1177/0954405419842412.

5. Morse EP and Srinivasan V. Size tolerancing revisited: A basic notion and its evolution in standards. Proceedings of the Institution of Mechanical Engineers, Part B: Journal of Engineering Manufacture 2013; 227: 662-671. DOI: 10.1177/0954405412470418.

6. Salomons OW, Poerink HJJ, Haalboom FJ, et al. A computer aided tolerancing tool I: Tolerance specification. Computers in Industry 1996; 31: 161-174. DOI: 10.1016/0166-3615(96)00046-2.

7. Cao Y and Srinivasan V. Special Issue: Geometric Tolerancing. Journal of Computing and Information Science in Engineering 2015; 15: 020301-020301-020301. DOI: 10.1115/1.4030073.

8. $\mathrm{Wu} \mathrm{Y}$. The correlational design method of the dimension tolerance and geometric tolerance for applying material conditions. The International Journal of Advanced Manufacturing Technology 2018; 97: 1697-1710. DOI: 10.1007/s00170-018-2052-4.

9. Clément A, Rivière A, Serré P, et al. The TTRS: 13 Constraints for Dimensioning and Tolerancing. 1998, p.122-129.

10. Gunay EE and Kula U. A two-stage stochastic rule-based model to determine pre-assembly buffer content. Journal of Industrial Engineering International 2018; 14: 655-663.

11. Selva D, Cameron B and Crawley EF. A rule-based method for scalable and traceable evaluation of system architectures. Research in Engineering Design 2014; 25: 325-349. DOI: 10.1007/s00163-014-0180-x.

12. Zhang $\mathrm{Y}$, Li Z, Gao J, et al. New reasoning algorithm for assembly tolerance specifications and corresponding tolerance zone types. Computer-Aided Design 2011; 43: 1606-1628. DOI: 10.1016/j.cad.2011.06.008.

13. Zhong Y, Qin Y, Huang M, et al. Automatically generating assembly tolerance types with an ontology-based approach. Computer-Aided Design 2013; 45: 1253-1275. DOI: 10.1016/j.cad.2013.06.006.

14. Qin Y, Zhong Y, Huang M, et al. An assembly tolerance representation model based on spatial relations for generating assembly tolerance types. Proceedings of the Institution of Mechanical Engineers, Part C: Journal of Mechanical Engineering Science 2014; 228: 1005-1020. DOI: 10.1177/0954406213495501.

15. $\mathrm{Hu} \mathrm{J}$, Xiong $\mathrm{G}$ and $\mathrm{Wu} \mathrm{Z}$. A variational geometric constraints network for a tolerance types specification. The International Journal of Advanced Manufacturing Technology 2004; 24: 214-222. DOI: 10.1007/s00170-003-1572-7.

16. Anselmetti B and Mawussi K. Computer Aided Tolerancing Using Positioning Features. Journal of Computing and Information Science in Engineering 2003; 3: 15. DOI: 10.1115/1.1565074.

17. Anselmetti B, Chavanne R, Yang J-X, et al. Quick GPS: A new CAT system for single-part tolerancing. Computer-Aided Design 2010; 42: 768-780. DOI: 10.1016/j.cad.2010.04.006.

18. Cao Y, Zhang H, Li B, et al. Study on functional specification scheme on interface based on positioning features. Proceedings of the Institution of Mechanical Engineers, Part B: Journal of Engineering Manufacture 2013; 227: 745-753. DOI: 10.1177/0954405413479112. 
19. Armillotta A. A method for computer-aided specification of geometric tolerances. Computer-Aided Design 2013; 45: 1604-1616. DOI: 10.1016/j.cad.2013.08.007.

20. Haghighi P, Mohan P, Kalish N, et al. Toward Automatic Tolerancing of Mechanical Assemblies: First-Order GD\&T Schema Development and Tolerance Allocation. Journal of Computing and Information Science in Engineering 2015; 15: 041003-041003-041009. DOI: 10.1115/1.4030939.

21. Nam, J., Jo, N., Kim, J. S., et al. Development of a health monitoring and diagnosis framework for fused deposition modeling process based on a machine learning algorithm. Proceedings of the Institution of Mechanical Engineers, Part B: Journal of Engineering Manufacture 2019; DOI:10.1177/0954405419855224.

22. Cao Y, Zhao Q, Liu T, et al. The Strategy of Datum Reference Frame Selection Based on Statistical Learning. Journal of Computing and Information Science in Engineering 2018; 18: 21002. DOI: 10.1115/1.4039380.

23. Qin Y, Lu W, Qi Q, et al. Towards an ontology-supported case-based reasoning approach for computer-aided tolerance specification. Knowledge-Based Systems 2018; 141: 129-147. DOI: 10.1016/j.knosys.2017.11.013.

24. Humienny $\mathrm{Z}$ and Berta $\mathrm{M}$. Interactive multimedia learning environment for geometrical specification indication \& verification rules. Procedia CIRP 2018; 75: 161-166. DOI: 10.1016/j.procir.2018.03.332.

25. Goetz S, Schleich B and Wartzack S. A new approach to first tolerance evaluations in the conceptual design stage based on tolerance graphs. Procedia CIRP 2018; 75: 167-172. DOI: https://doi.org/10.1016/j.procir.2018.04.030.

26. Patalano S, Vitolo F, Gerbino S, et al. A graph-based method and a software tool for interactive tolerance specification. Procedia CIRP 2018; 75: 173-178. DOI: https://doi.org/10.1016/j.procir.2018.04.077.

27. Wang QQ, He Z, Zhu Z, et al. Evaluations of cellulose accessibilities of lignocelluloses by solute exclusion and protein adsorption techniques. Biotechnology and Bioengineering 2012; 109: 381-389. DOI: 10.1002/bit.23330.

28. Al Hage J, AitTmazirte N, El Najjar ME, et al. Fault detection and exclusion method for a tightly coupled localization system. In: 2015 pp.623-628. IEEE.

29. Suh H. Axiomatic Design: advances and applications MIT-Pappalardo series in Mechanical Engineering. Oxford University Press, USA, 2001.

30. Liang $\mathrm{J}$ and Shi Z. THE INFORMATION ENTROPY, ROUGH ENTROPY AND KNOWLEDGE GRANULATION IN ROUGH SET THEORY. International Journal of Uncertainty, Fuzziness and Knowledge-Based Systems 2004; 12: 37-46. DOI: 10.1142/S0218488504002631.

31. Weißgerber M, Ebermann M, Gröger S, et al. Requirements for Datum Systems in Computer Aided Tolerancing and the Verification Process. Procedia CIRP 2016; 43: 238-243. DOI: https://doi.org/10.1016/j.procir.2016.02.096.

32. Srinivasan V, Shakarji CM and Morse EP. On the Enduring Appeal of Least-Squares Fitting in Computational Coordinate Metrology. Journal of Computing and Information Science in Engineering 2012; 12: 11008. DOI: $10.1115 / 1.3647877$.

33. Srinivasan V. Reflections on the role of science in the evolution of dimensioning and tolerancing standards. Proceedings of the Institution of Mechanical Engineers, Part B: Journal of Engineering Manufacture 2012; 227: 3-11. DOI: 10.1177/0954405412464012.

34. Zhao Q, Cao Y, Liu T, et al. Tolerance specification of the plane feature based on the axiomatic design. Proceedings of the Institution of Mechanical Engineers, Part C: Journal of Mechanical Engineering Science 2018; 233: 1481-1492. DOI: 10.1177/0954406218772001.

35. Yuan X, Li A, Shen Y, et al. Kinematic path analysis of kinematically indeterminate systems. KSCE Journal of Civil Engineering 2016; 20: 813-819. DOI: 10.1007/s12205-015-0481-2.

36. Cao Y, Liu T, Yang J, et al. A novel tolerance analysis method for three-dimensional assembly. Proceedings 
of the Institution of Mechanical Engineers, Part B: Journal of Engineering Manufacture 2018; 233: 1818-1827. DOI: $10.1177 / 0954405418789979$.

37. Peng P, Wang J. NOSCNN: A robust method for fault diagnosis of RV reducer. Measurement 2019; 138 : 652-658. https://doi.org/10.1016/j.measurement.2019.02.080.

38. Durupt, A., Matthieu B., Sébastien R., et al. An Extended Framework for Knowledge Modelling and Reuse in Reverse Engineering Projects. Proceedings of the Institution of Mechanical Engineers, Part B: Journal of Engineering Manufacture 2019; https://doi.org/10.1177/0954405418789973.

39. Kang, P. G., Woo, Y. Y. and Moon, Y. H.. Effects of circular holes on the web warping of perforated blanks in flexible roll forming. Proceedings of the Institution of Mechanical Engineers, Part B: Journal of Engineering Manufacture 2019; https://doi.org/10.1177/0954405418805666.

40. Jain, R., Pal, S. K., \& Singh, S. B.. Investigation on effect of pin shapes on temperature, material flow and forces during friction stir welding: A simulation study. Proceedings of the Institution of Mechanical Engineers, Part B: Journal of Engineering Manufacture 2019; https://doi.org/10.1177/0954405418805615.

41. JJiang, L., Li, Y., Han, L., et al. A workpiece registration and localization adjustment method with contact inspection under multi-tolerance conditions. Proceedings of the Institution of Mechanical Engineers, Part B: Journal of Engineering Manufacture 2018; https://doi.org/10.1177/0954405418789978. 


\section{Annex}

Table A1 Possible geometrical tolerances used for the feature of different geometrical types

\begin{tabular}{|c|c|c|}
\hline Geometrical Type & Possible geometrical tolerances & Example (see Fig. 7) \\
\hline Plane* & $\begin{array}{c}D P_{1}(-), D P_{2}(\square), D P_{5}(/ /), D P_{6}(\perp), \\
D P_{7}(\measuredangle), D P_{9}(\overline{=}), D P_{10}(\phi), D P_{11}(/) \\
\text { or } D P_{12}(\measuredangle)\end{array}$ & Feature f4, cg-2 \\
\hline Revolving surface* & 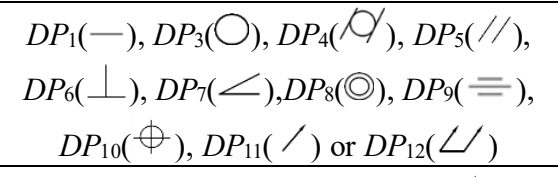 & Feature f3, cg-3 \\
\hline Sphere* & $D P_{3}(\bigcirc)$ and $D P_{13}(\bigcirc)$; or $D P_{10}(\phi)$ & 1 \\
\hline Pattern** & $D P_{10}(\phi)$ & Feature cg-4, cg-6 \\
\hline Key Slot/Multiple & Axial: $D P_{9}(\overline{=})$ & Feature $\mathrm{f} 2$ \\
\hline keys ** & Radial: $D P_{11}(/)$ & \\
\hline Gear teeth** & $D P_{11}(/)$ & Feature cg-7 \\
\hline Free curve $* *$ & $D P_{14}(\cap)$ & / \\
\hline Free form surface $* *$ & $D P_{15}(\bigcirc)$ & I \\
\hline
\end{tabular}

Note: * If the number of possible geometrical tolerances is more significant than one, then the selection will be carried out. For example, the geometrical type of plane, there are nine possible geometrical tolerances (see Table A1).

** The number of possible geometrical tolerances is equal to one, then the selection is made as there are only one results (i.e. no selection needed). For example, the geometrical type of Pattern, Free curve, Freeform surface (see Table A1).

Table A2 Possible selection schemes for different positions in relation to the primary datum

\begin{tabular}{|c|c|c|}
\hline $\begin{array}{l}\text { Position relation with } \\
\text { primary datum }\end{array}$ & Selection scheme for planes & $\begin{array}{l}\text { Selection scheme for revolving } \\
\text { features }\end{array}$ \\
\hline Primary datum & $D P_{1}(-), D P_{2}(\square)$ & $D P_{1}(-), D P_{3}(\bigcirc), D P_{4}(d /)$ \\
\hline Parallelism & $\begin{array}{c}D P_{1}(-), D P_{2}(\square), D P_{5}(/ /) \\
\quad \text { or } D P_{10}(\phi)\end{array}$ & $\begin{array}{c}D P_{1}(-), D P_{3}(\bigcirc), D P_{4}(\phi /), D P_{5} \\
(/ /), D P_{10}(\phi)\end{array}$ \\
\hline Perpendicular & $\begin{array}{c}D P_{1}(-), D P_{2}(\square), D P_{6} \\
(\perp), D P_{10}(\phi), D P_{11}(/) \text { or } \\
D P_{12}(\llcorner)\end{array}$ & $\begin{array}{c}D P_{1}(-), D P_{3}(\bigcirc), D P_{4}(\phi), D P_{6} \\
(\perp), D P_{10}(\phi)\end{array}$ \\
\hline Angular & $\begin{array}{c}D P_{1}(-), D P_{2}(\square), D P_{7}(\measuredangle) \\
\quad \text { or } D P_{10}(\phi)\end{array}$ & $\begin{array}{c}D P_{1}(-), D P_{3}(\bigcirc), D P_{4}(\phi), D P_{7} \\
(\angle) \text { or } D P_{10}(\phi)\end{array}$ \\
\hline Coplanar & $\begin{array}{c}D P_{1}(-), D P_{2}(\square) \text { or } D P_{10} \\
(\phi)\end{array}$ & / \\
\hline Coaxial & I & $\begin{array}{c}D P_{1}(-), D P_{3}(\bigcirc), D P_{4}(\mathrm{~d} /), D P_{8} \\
\text { (@), } D P_{11}(/) \text { or } D P_{12}(\triangle)\end{array}$ \\
\hline Symmetry & $\begin{array}{c}D P_{1}(-), D P_{2}(\square) \text { or } \\
D P_{9}(=)\end{array}$ & $\begin{array}{c}D P_{1}(-), D P_{3}(\bigcirc), D P_{4}(d /), D P_{5} \\
(/ /), D P_{11}(\perp), D P_{12}(\angle) \text { or } D P_{9} \\
(\overline{=})\end{array}$ \\
\hline
\end{tabular}

